\title{
Dexmedetomidine Oral Mucosa Patch for Sedation Suppresses Apoptosis in Hippocampus of Normal Rats
}

\author{
Je Hoon Park ${ }^{1}$, Il Gyu Ko ${ }^{2}$, Sung Eun Kim² , Jun Jang Jin², Lakkyong Hwang², Chang Ju Kim², Soo Hwan Yoon³ Jongki Hong \\ Jun Young Chung ${ }^{4}$, Deok Won Lee ${ }^{5}$ \\ 'Department of Surgery, International St. Mary's Hospital, College of Medicine, Catholic Kwandong University, Incheon, Korea \\ ${ }^{2}$ Department of Physiology, College of Medicine, Kyung Hee University, Seoul, Korea \\ ${ }^{3}$ College of Pharmacy, Kyung Hee University, Seoul, Korea \\ ${ }^{4}$ Department of Anesthesiology and Pain Medicine, Kyung Hee University Hospital at Gangdong, College of Medicine, Kyung Hee University, Seoul, Korea \\ ${ }^{5}$ Department of Oral and Maxillofacial Surgery, Kyung Hee University Dental Hospital at Gangdong, College of Dentistry, Kyung Hee University, Seoul, Korea
}

Purpose: Dexmedetomidine, an a2-adrenergic agonist, provides sedative and analgesic effects without significant respiratory depression. Dexmedetomidine has been suggested to have an antiapoptotic effect in response to various brain insults. We developed an oral mucosa patch using dexmedetomidine for sedation. The effects of the dexmedetomidine oral mucosa patch on cell proliferation and apoptosis in the hippocampus were evaluated.

Methods: A hydrogel oral mucosa patch was adhered onto the oral cavity of physiologically normal rats, and was attached for 2 hours, 6 hours, 12 hours, or 24 hours. Plasma dexmedetomidine concentrations were determined by liquid chromatography-electrospray ionization-tandem mass spectrometry-multiple-ion reaction monitoring (LC-ESI-MS/MS-MRM). Cell proliferation in the hippocampus was detected by Ki-67 immunohistochemistry. Caspase- 3 immunohistochemistry, terminal deoxynucleotidyl transferase-mediated dUTP nick end labeling staining, and Western blotting for Bax and Bcl-2 were performed to detect hippocampal apoptosis. The levels of brain-derived neurotrophic factor (BDNF) and tyrosine kinase B (TrkB) in the hippocampus were also measured by Western blotting.

Results: Plasma dexmedetomidine concentration increased according to the attachment time of the dexmedetomidine oral mucosa patch. Hippocampal cell proliferation did not change due to the dexmedetomidine oral mucosa patch, and the dexmedetomidine oral mucosa patch exerted no significant effect on BDNF or TrkB expression. In contrast, the dexmedetomidine oral mucosa patch exerted an antiapoptotic effect depending on the attachment time of the dexmedetomidine oral mucosa patch.

Conclusions: A dexmedetomidine oral mucosa patch can be used as a convenient tool for sedation, and is of therapeutic value due to its antiapoptotic effects under normal conditions.

Keywords: Dexmedetomidine; Apoptosis; Cell proliferation; Hippocampus; Rats

- Fund/Grant Support: This study was supported by a grant from the National Research Foundation of Korea (NRF-2016R1A2B4014600).

- Research Ethics: We followed the guidelines of the Institutional Care and Use Committee of Kyung Hee University during all laboratory procedures (KHUASP[SE]-16-021), and all experiments were performed in accordance with the guiding principles for the care and use of animals approved by the Council of the National Institutes of Health Guide for the Care and Use of Laboratory Animals.

- Conflict of Interest: No potential conflict of interest relevant to this article was reported.

Corresponding author: Deok Won Lee (iD http://orcid.org/0000-0001-6233-1436 Department of Oral and Maxillofacial Surgery, Kyung Hee University Dental Hospital at Gangdong, School of Dentistry, Kyung Hee University, 892 Dongnam-ro, Gangdong-gu, Seoul 05278, Korea

E-mail: verycutebear@hanmail.net / Tel: +82-2-440-6172 / Fax: +82-2-440-7525

Submitted: April 1, 2017 / Accepted after revision: April 18, 2017 


\author{
- HIGHLIGHTS \\ - We developed an oral mucosa patch using dexmedetomidine for se- \\ dation. \\ - This oral mucosa patch continuously delivered dexmedetomidine into \\ the blood. \\ - Dexmedetomidine oral mucosa patch exerted an antiapoptotic effect.
}

\section{INTRODUCTION}

Dexmedetomidine is an a2-adrenergic agonist, and it provides sedative and analgesic effects without significant respiratory depression. It has been used to promote postoperative and intensive care sedation and analgesia. The sedative effect of dexmedetomidine has led to the emergence of several interesting applications in the anesthetic field, with benefits in the perioperative context. However, dexmedetomidine was approved for sedation in the intensive care unit in the United States in 1999, and its application as a form of anesthesia remains an off-label use. Further studies are needed to establish the role of dexmedetomidine in the perioperative period [1].

Anesthetic agents generally exert detrimental effects on the brain, causing apoptotic neurodegeneration and inducing learning and memory impairments [2]. Ketamine and isoflurane initiate apoptosis and impair brain function in the developing brain of rats; in contrast, dexmedetomidine is known to attenuate ketamine and isoflurane-induced neurotoxicity $[3,4]$. Dexmedetomidine has been suggested to have neuroprotective, cardioprotective, and renoprotective effects [5].

Dexmedetomidine exerts a protective effect against cerebral ischemia-induced injury by enhancing the expression of the antiapoptotic proteins $\mathrm{Bcl}-2$ and $\mathrm{Mdm}-2$ [6]. A preconditioning effect of dexmedetomidine on oxygen and glucose deprivation in hippocampal slices has been reported [7]. Dexmedetomidine was found to ameliorate isoflurane-induced injury in the brain of developing rats [8], and also inhibited staurosporine- or wortmannin-induced injury in cortical neuronal cultures [9]. Kim et al. [10] reported that dexmedetomidine increased the amplitude of evoked field excitatory postsynaptic potentials in oxygen- and glucose-deprived hippocampal slices. Dexmedetomidine exerted an antiapoptotic effect in rats with intracerebral hemorrhage [11] and in gerbils with cerebral ischemia [12].

Dexmedetomidine is widely used for patients in surgical and nonsurgical intensive care units. However, the intravenous administration of dexmedetomidine is cumbersome and inconvenient for clinical applications. The instability of administration through the nasal mucosa is also a limitation to the clinical ap- plication of dexmedetomidine. In particular, the use of safe sedatives in the dental field or in urological and gynecological procedures that give patients vague fears is necessary. In order to avoid the fear and inconvenience associated with injections, we developed an oral mucosa patch for sedation using dexmedetomidine.

In this study, we evaluated the effects of the dexmedetomidine oral mucosa patch on cell proliferation and apoptosis in the hippocampus. For this purpose, immunohistochemistry for Ki-67 and caspase-3 and terminal deoxynucleotidyl transferasemediated dUTP nick end labeling (TUNEL) staining were conducted. Western blotting was performed to assess levels of Bax, $\mathrm{Bcl}-2$, brain-derived neurotrophic factor (BDNF), and tyrosine kinase B (TrkB). Plasma dexmedetomidine concentrations according to the attachment time of the oral mucosa patch were also determined.

\section{MATERIALS AND METHODS}

\section{Preparation of Oral Mucosa Patch for Sedation}

Polyacrylic acid ( $3 \%$ by weight, $100 \mathrm{~mL}$ ) was prepared by dissolving polyacrylic acid (molecular weight, 450,000 g/mol; $3 \mathrm{~g}$ ) in distilled water $(97 \mathrm{~mL})$. The aqueous solution of polyacrylic acid was poured into a petri dish with a diameter of $1 \mathrm{~cm}$ to a thickness of $3 \mathrm{~mm}$, sealed with a polyethylene film, and irradiated with $10 \mathrm{kGy}$ of radiation to prepare a crosslinked polyacrylic acid hydrogel. The prepared hydrogel was dried in an oven at $37^{\circ} \mathrm{C}$ for 24 hours, cut into a diameter of $12 \mathrm{~mm}$ (weight, $0.04 \mathrm{~g} ; 7 \%$ by weight), and then immersed in a sieve with 0.5 $\mathrm{mL}$ of dexmedetomidine $(200 \mu \mathrm{g} / 2 \mathrm{~mL} / \mathrm{vial})$ for 24 hours. The drug-loaded hydrogel was dried in an oven at $37^{\circ} \mathrm{C}$ for 24 hours to prepare an oral mucoadhesive patch for sedation (Fig. 1).

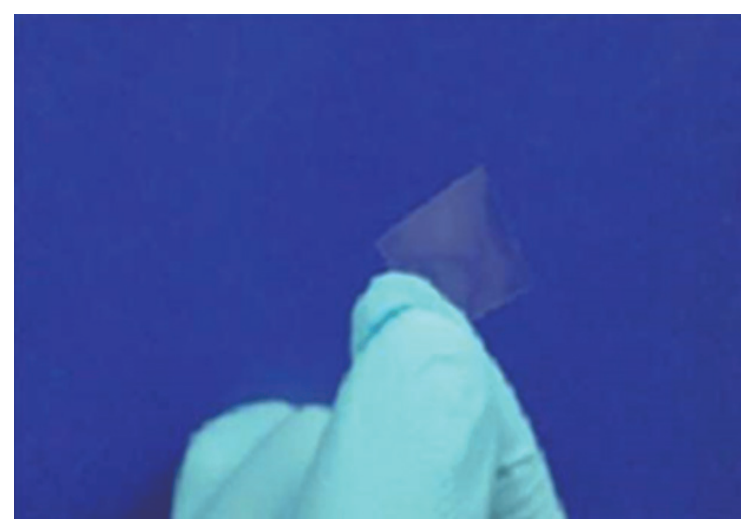

Fig. 1. The sedative hydrogel oral mucosa patch. 


\section{Animals and Treatments}

For this experiment, male Sprague-Dawley rats (weighing $350 \pm 10 \mathrm{~g}, 15$ weeks old) were used, and the experimental procedures were conducted according to the animal care guidelines of the National Institutes of Health and the Korean Academy of Medical Sciences. The rats were randomly divided into 4 groups ( $\mathrm{n}=8$ in each group): a control group, and groups in which the dexmedetomidine patch was attached for 2, 6, 12, and 24 hours, respectively.

\section{Application of the Dexmedetomidine Oral Mucosa Patch} After performing anesthesia with Zoletil $50(10 \mathrm{mg} / \mathrm{kg}$, intraperitoneally; Vibac Laboratories, Carros, France), the rats were placed in the supine position. The oral mucosa patch was then adhered onto the oral cavity of the rats (Fig. 2). After 2 hours, 6 hours, 12 hours, or 24 hours of attachment (depending on the treatment group), blood sampling via heart puncture was performed.

\section{Determination of Plasma Dexmedetomidine Concentration by Liquid Chromatography-Tandem Mass Spectrometry}

Plasma was collected from each group and a stock solution was prepared by dissolving $1 \mathrm{mg}$ of a dexmedetomidine standard in $1 \mathrm{~mL}$ of methanol, and then diluting it with 50\% methanol in water $(v / v)$ when necessary. Plasma samples $(200 \mu \mathrm{L})$ were extracted by liquid-liquid extraction. Dexmedetomidine in plasma was extracted with $1 \mathrm{~mL}$ ethyl acetate, with thorough shaking for 5 minutes. The ethyl acetate layer was passed through a membrane filter and then dried under a gentle nitrogen stream. The dried residue was redissolved in $200 \mu \mathrm{L}$ of $50 \%$ methanol in water $(\mathrm{v} / \mathrm{v})$ and centrifuged at $13,000 \mathrm{rpm}$ for 5 minutes. The sample supernatant was injected into liquid chromatographyelectrospray ionization-tandem mass spectrometry-multiple- ion reaction monitoring (LC-ESI-MS/MS-MRM).

All liquid chromatography-tandem mass spectrometry analyses were performed with an Agilent 1200 series high-performance liquid chromatography system coupled with an API 3200 mass spectrometer (MDS Sciex, Concord, ON, Canada). The chromatographic separation was carried out with a Kintex XB C18 column ( $100 \mathrm{~mm} \times 2.1 \mathrm{~mm}$; internal diameter, $2.6 \mu \mathrm{m}$; Phenomenex, Torrance, CA, USA). A binary gradient separation was performed using a flow rate of $0.2 \mathrm{~mL} / \mathrm{min}$ using mobile phases $\mathrm{A}$ ( $0.1 \%$ formic acid in water) and $\mathrm{B}$ (acetonitrile). The gradient profile was $0-2$ minutes held at $15 \% \mathrm{~B}$, a 2- to 3-minute linear increase in B from 15\% to $97 \%$, 3-6 minutes held at $97 \%$ B; and 6-10 minutes (post acquisition time) starting with a mobile phase of $15 \% \mathrm{~B}$ to re-equilibrate the column. The total run time for each injection was 10 minutes, and the injection volume was $5 \mu \mathrm{L}$. The mass spectrometer was run in the positive-ion mode of the electrospray ionization source, and the mass spectrometric conditions were as follows: curtain gas, 20 psi; electron voltage, $5,500 \mathrm{~V}$; temperature, $500^{\circ} \mathrm{C}$; nebulizing gas, 50 psi; and heating gas, 50 psi.

For the quantification analysis, the multiple-ion reaction monitoring (MRM) mode was applied using 2 characteristic MRM transitions, such as the quantitative ion and the confirmation ion. The selection criteria of the product ions were established to choose the 2 most intense ions, in order to increase the specificity of the product ions and to minimize the background signal.

\section{Tissue Preparation}

The rats were sacrificed immediately after blood sampling. The rats received a transcardial injection of $50 \mathrm{mM}$ phosphate-buffered saline (PBS), and they were then fixed with a freshly prepared solution consisting of $4 \%$ paraformaldehyde in $100 \mathrm{mM}$
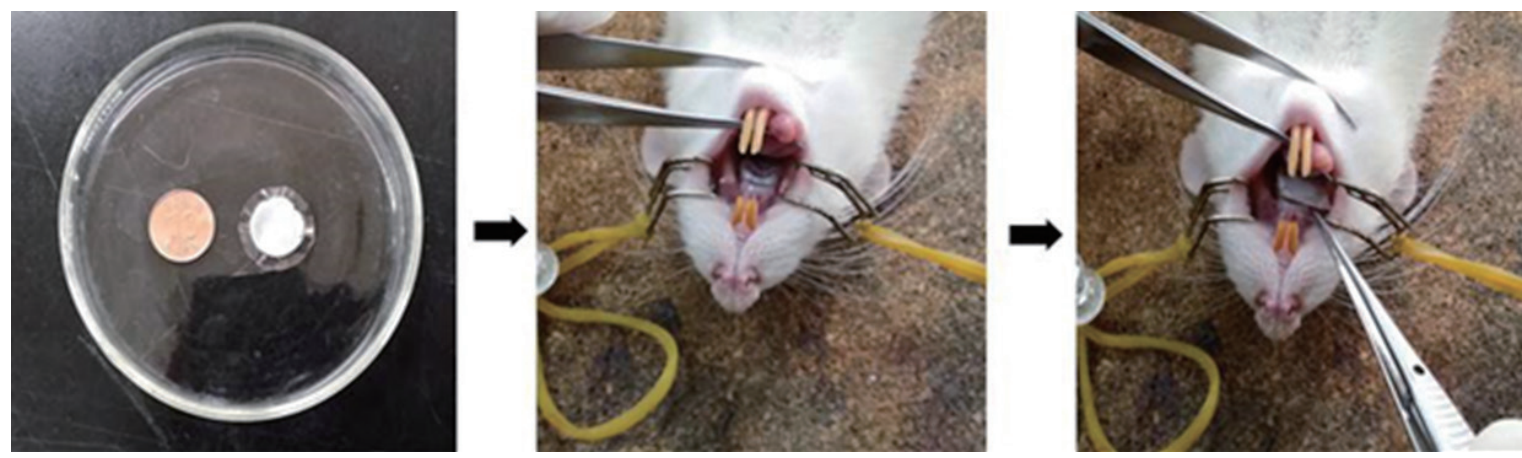

Fig. 2. The procedure of oral mucosa patch application. 
phosphate buffer ( $\mathrm{pH}, 7.4)$. The rats' brains were dissected and stored overnight in the same fixative solution, and then they were immersed into a $30 \%$ sucrose solution for cryoprotection. Subsequently, $40-\mu \mathrm{m}$-thick slices were coronally sectioned using a cryostat (Leica, Nussloch, Germany). On average, 10 hippocampal slice sections, from 2.5 to $2.7 \mathrm{~mm}$ posterior from the bregma, were collected from each rat.

\section{Ki-67 Immunohistochemistry}

Ki-67 immunohistochemistry was performed according to a previously described method $[13,14]$. The sections were incubated in $0.05 \mathrm{M}$ PBS for 10 minutes and then washed 3 times with $50 \mathrm{mM}$ PBS. The sections were incubated in $3 \% \mathrm{H}_{2} \mathrm{O}_{2}$ for 30 minutes, and then incubated overnight with mouse antiKi-67 antibody (1:500; Novocastra Laboratories, Newcastle, UK). The next day, the sections were incubated with biotinylated anti-mouse secondary antibody (Vector Laboratories, Burlingame, CA, USA) for 1 hour. The sections were subsequently incubated with an avidin-biotin-peroxidase complex (Vector Laboratories) at room temperature for 1 hour. Immunoreactivity was visualized by incubating the sections in a solution consisting of $0.05 \%$ diaminobenzidine tetrahydrochloride (DAB) and $0.01 \% \mathrm{H}_{2} \mathrm{O}_{2}$ in $50 \mathrm{mM}$ Tris buffer $(\mathrm{pH}, 7.6)$ for approximately 3 minutes. After the sections were mounted on gelatincoated glass slides, the coverslips were mounted using Permount (Fisher Scientific, New Jersey, NJ, USA).

\section{Caspase-3 Immunohistochemistry}

Immunohistochemistry for caspase-3 was performed according to previously described methods $[15,16]$. The sections were incubated overnight with caspase-3 antibody (1:500; Santa Cruz Biotechnology, Santa Cruz, CA, USA). The next day, the sections were incubated with biotinylated mouse secondary antibody (1:200; Vector Laboratories) for 1 hour. The secondary antibody was amplified with a Vector Elite ABC kit (1:100; Vector Laboratories). Antibody-biotin-avidin-peroxidase complexes were visualized using $0.03 \% \mathrm{DAB}$. The sections were mounted onto gelatin-coated slides, and the coverslips were mounted using Permount (Fisher Scientific).

\section{TUNEL Staining}

TUNEL staining was performed using an In Situ Cell Death Detection Kit (Roche, Mannheim, Germany), according to previously described methods $[15,17]$. The sections were postfixed in ethanol-acetic acid (2:1), and then were rinsed. The sections were then incubated with proteinase $\mathrm{K}(100 \mu \mathrm{g} / \mathrm{mL})$, rinsed, incubated in $3 \% \mathrm{H}_{2} \mathrm{O}_{2}$, and permeabilized with $0.5 \%$ Triton X-100. After the sections were rinsed again, the sections were incubated in the TUNEL reaction mixture. After the sections were rinsed, the sections were visualized using ConverterPOD with $0.03 \%$ DAB. Mayer hematoxylin (DAKO, Glostrup, Denmark) was used as a counterstain. The sections were mounted onto gelatin-coated slides, and the coverslips were mounted using Permount (Fisher Scientific).

\section{Western Blotting for Bax and Bcl-2}

Western blot analysis was performed to determine the expression of Bax and Bcl-2, according to previously described methods $[15,17]$. Hippocampi were dissected and collected, and hippocampal tissues were immediately frozen at $-70^{\circ} \mathrm{C}$. The hippocampal tissues were homogenized on ice, and they were lysed in a lysis buffer containing 50mM N-2-hydroxyethylpiperazine-N2-ethanesulfonic acid (pH, 7.5), $150 \mathrm{mM} \mathrm{NaCl}, 10 \%$ glycerol, $1 \%$ Triton X-100, 1mM phenylmethylsulfonyl fluoride, $1 \mathrm{mM}$ ethyleneglycol-bis-(b-aminoethylether)-N,N,N',N'-tetraacetic acid, $1.5 \mathrm{mM} \mathrm{MgCl}_{2} \cdot 6 \mathrm{H}_{2} \mathrm{O}, 1 \mathrm{mM}$ sodium ortho-vanadate, and $100 \mathrm{mM}$ sodium fluoride. Protein content was measured using a Bio-Rad colorimetric protein assay kit (Bio-Rad, Hercules, CA, USA). Protein samples $(30 \mu \mathrm{g})$ were separated on sodium dodecyl sulfate-polyacrylamide gel and transferred onto a nitrocellulose membrane. The membranes were incubated with 5\% skim milk in Tris-buffered saline containing $0.1 \%$ Tween-20. The membranes were then incubated at $4^{\circ} \mathrm{C}$ with the following primary antibodies overnight: mouse anti- $\beta$-actin, mouse antiBcl-2, and mouse anti-Bax (1:1,000; Santa Cruz Biotechnology). Subsequently, the membranes were incubated with following secondary antibodies for 1 hour: horseradish peroxidase conjugated anti-mouse antibody for Bax and Bcl-2 (1:3,000; Vector Laboratories) and horseradish peroxidase-conjugated antimouse antibody for $\beta$-actin (1:2,000; Vector Laboratories). An enhanced chemiluminescence detection kit (Santa Cruz Biotechnology) was used for band detection.

\section{Western Blotting for BDNF and TrkB}

Western blot analysis was conducted to characterize the expression of BDNF and TrkB, according to previously described methods [12,14]. Mouse $\beta$-actin antibody (1:1,000; Santa Cruz Biotechnology), rabbit BDNF antibody (1:500; Santa Cruz Biotechnology), and rabbit TrkB antibody (1:1,000; Santa Cruz Biotechnology) were used as primary antibodies. The second- 
ary antibodies were horseradish peroxidase conjugated antirabbit antibody for BDNF and TrkB (1:3,000; Vector Laboratories) and horseradish peroxidase-conjugated anti-mouse antibody for $\beta$-actin (1:2,000; Vector Laboratories). An enhanced chemiluminescence detection kit (Santa Cruz Biotechnology) was used for band detection.

\section{Data Analysis}

In the selected samples of the hippocampal dentate gyrus, the numbers of Ki-67-positive, TUNEL-positive, and caspase-3positive cells were counted hemilaterally using a light microscope (Olympus, Tokyo, Japan). The area of the hippocampal dentate gyrus was measured using the Image-Pro Plus computer-assisted image analysis system (Media Cybernetics Inc., Silver Spring, MD, USA). These values were expressed as the numbers of cells per square millimeter in the hippocampal dentate gyrus. The detected bands were calculated densitometrically to characterize Bax, Bcl-2, BDNF, and TrkB expression using the Image-Pro Plus image analysis system (Media Cybernetics Inc.). Statistical analysis was performed with 1-way analysis of variance followed by the Duncan post hoc test. The results are expressed as the mean \pm standard error of the mean, and statistical significance was set at $\mathrm{P}<0.05$.

\section{RESULTS}

\section{Analysis of Plasma Dexmedetomidine by LC-ESI-MS/MS- MRM}

Plasma dexmedetomidine concentrations increased according to attachment time, reached a maximum level at 12 hours after attachment, and then rapidly decreased at 24 hours after attachment (Table 1).

\section{Effect of Dexmedetomidine on Cell Proliferation in the Hippocampal Dentate Gyrus}

Cell proliferation in the hippocampal dentate gyrus was not significantly associated with the attachment time of dexmedetomidine (Fig. 3).

Table 1. Plasma dexmedetomidine concentration in each group

\begin{tabular}{lcccc}
\hline \multirow{2}{*}{ Analyte } & \multicolumn{4}{c}{ Attachment time } \\
\cline { 2 - 5 } & 2 Hours & 6 Hours & 12 Hours & 24 Hours \\
\hline Dexmedetomidine & $\begin{array}{c}6.62 \\
\mathrm{ng} / \mathrm{mL}\end{array}$ & $\begin{array}{c}10.13 \\
\mathrm{ng} / \mathrm{mL}\end{array}$ & $\begin{array}{c}14.16 \\
\mathrm{ng} / \mathrm{mL}\end{array}$ & \begin{tabular}{c}
$\mathrm{ng} / \mathrm{mL}$ \\
\hline
\end{tabular}
\end{tabular}

\section{Effect of Dexmedetomidine on DNA Fragmentation in the Hippocampal Dentate Gyrus}

DNA fragmentation in the hippocampal dentate gyrus was inversely associated with the attachment time of dexmedetomidine (Fig. 4).

\section{Effect of Dexmedetomidine on Caspase-3 Expression in the Hippocampal Dentate Gyrus}

Caspase-3 expression in the hippocampal dentate gyrus was inversely associated with the attachment time of dexmedetomidine (Fig. 5).

\section{Effect of Dexmedetomidine on Bax and Bcl-2 Expression in the Hippocampus}

Bax expression did not change until 12 hours after the attach-
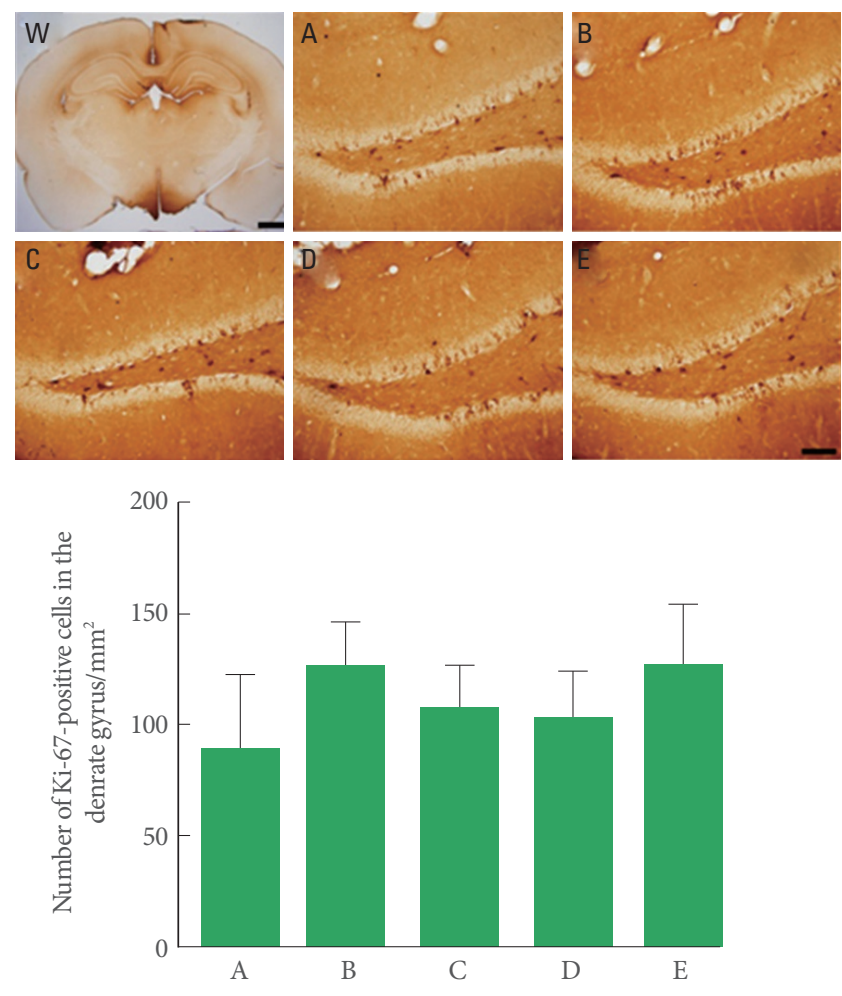

Fig. 3. Effects of dexmedetomidine on the number of Ki67-positive cells in the hippocampal dentate gyrus. Upper panel: photomicrographs of Ki-67-positive cells. The scale bar represents $50 \mu \mathrm{m}(\mathrm{W})$ and $150 \mu \mathrm{m}$ (A-E). Lower panel: The number of Ki-67-positive cells in each group. W, whole brain; A, control group; $\mathrm{B}$, the group in which dexmedetomidine was attached for 2 hours; $\mathrm{C}$, the group in which dexmedetomidine was attached for 6 hours; D, the group in which dexmedetomidine was attached for 12 hours; $\mathrm{E}$, the group in which dexmedetomidine was attached for 24 hours. 

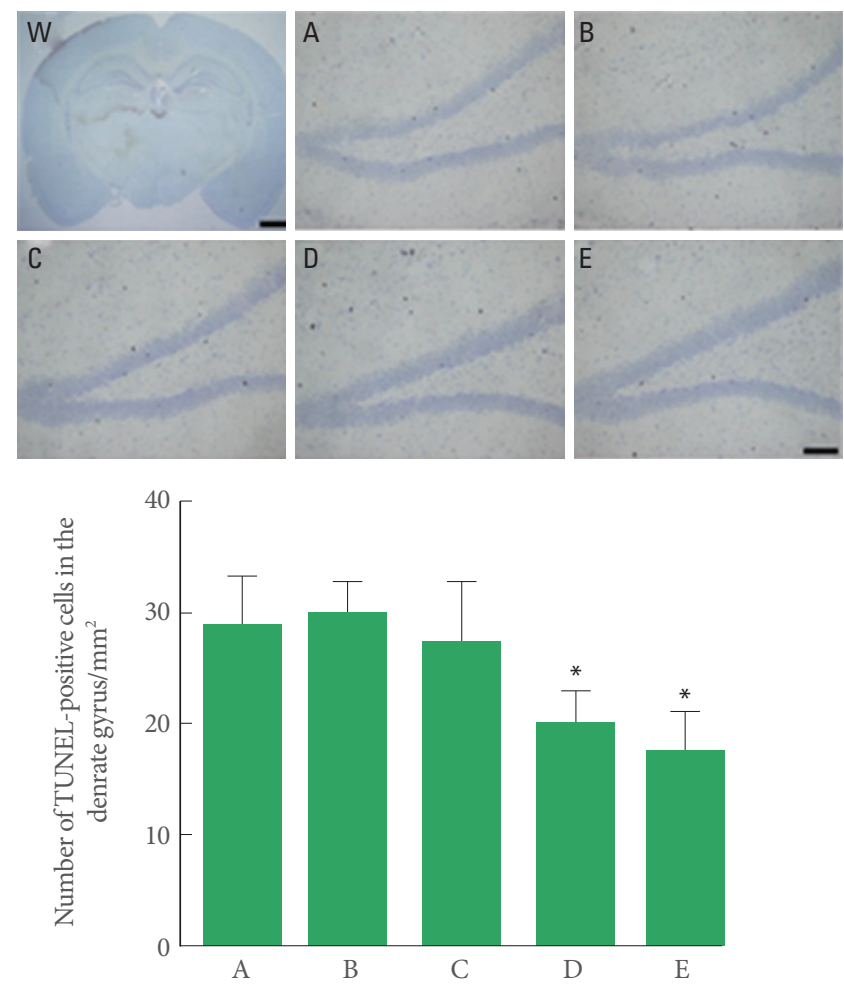

Fig. 4. The effect of dexmedetomidine on the number of terminal deoxynucleotidyl transferase-mediated dUTP nick end labeling (TUNEL)-positive cells in the hippocampal dentate gyrus. Upper panel: photomicrographs of TUNEL-positive cells. The scale bar represents $50 \mu \mathrm{m}(\mathrm{W})$ and $150 \mu \mathrm{m}(\mathrm{A}-\mathrm{E})$. Lower panel: the number of TUNEL-positive cells in each group. W, whole brain; A, control group; B, the group in which dexmedetomidine was attached for 2 hours; $\mathrm{C}$, the group in which dexmedetomidine was attached for 6 hours; $\mathrm{D}$, the group in which dexmedetomidine was attached for 12 hours; E, the group in which dexmedetomidine was attached for 24 hours. ${ }^{*} \mathrm{P}<0.05$ compared to the control group.

ment of dexmedetomidine; however, Bax expression decreased 24 hours after dexmedetomidine attachment (Fig. 6, lower left). Bcl-2 expression increased with the attachment time of dexmedetomidine (Fig. 6, lower middle). The ratio of Bax to Bcl-2 did not change until 12 hours after the attachment of dexmedetomidine; however, the ratio of Bax to $\mathrm{Bcl}-2$ decreased 24 hours after dexmedetomidine attachment (Fig. 6, lower right).

\section{Effect of Dexmedetomidine on BDNF and TrkB Expression in the HippocampusHippocampus}

BDNF expression did not vary with the attachment time of dexmedetomidine (Fig. 7, lower left). TrkB expression likewise did not vary with the attachment time of dexmedetomidine
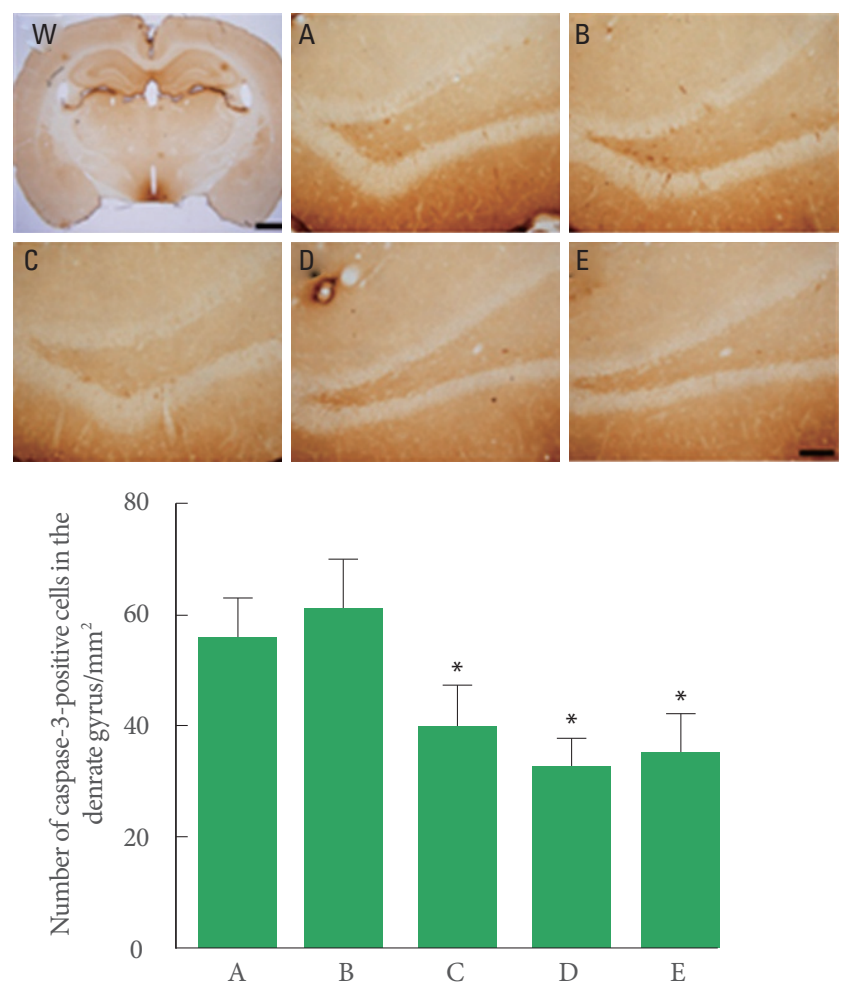

Fig. 5. Effect of dexmedetomidine on the number of caspase3-positive cells in the hippocampal dentate gyrus. Upper panel: photomicrographs of caspase-3-positive cells. The scale bar represents $50 \mu \mathrm{m}(\mathrm{W})$ and $150 \mu \mathrm{m}$ (A-E). Lower panel: the number of caspase-3-positive cells in each group. W, whole brain; A, control group; $\mathrm{B}$, the group in which dexmedetomidine was attached for 2 hours; $\mathrm{C}$, the group in which dexmedetomidine was attached for 6 hours; $D$, the group in which dexmedetomidine was attached for 12 hours; $\mathrm{E}$, the group in which dexmedetomidine was attached for 24 hours. ${ }^{*} \mathrm{P}<0.05$ compared to the control group.

(Fig. 7, lower right).

\section{DISCUSSION}

In addition to the antiapoptotic effect of dexmedetomidine associated with various brain insults $[11,12]$, Han et al. [18] demonstrated that dexmedetomidine did not induce apoptosis or degeneration when injected in the brachial plexus of normal rats. We determined the attachment time-dependent plasma concentrations of dexmedetomidine and evaluated the attachment time-dependent effects of this oral mucosa patch on cell proliferation and apoptosis in the hippocampus. In the present study, plasma dexmedetomidine concentrations increased ac- 

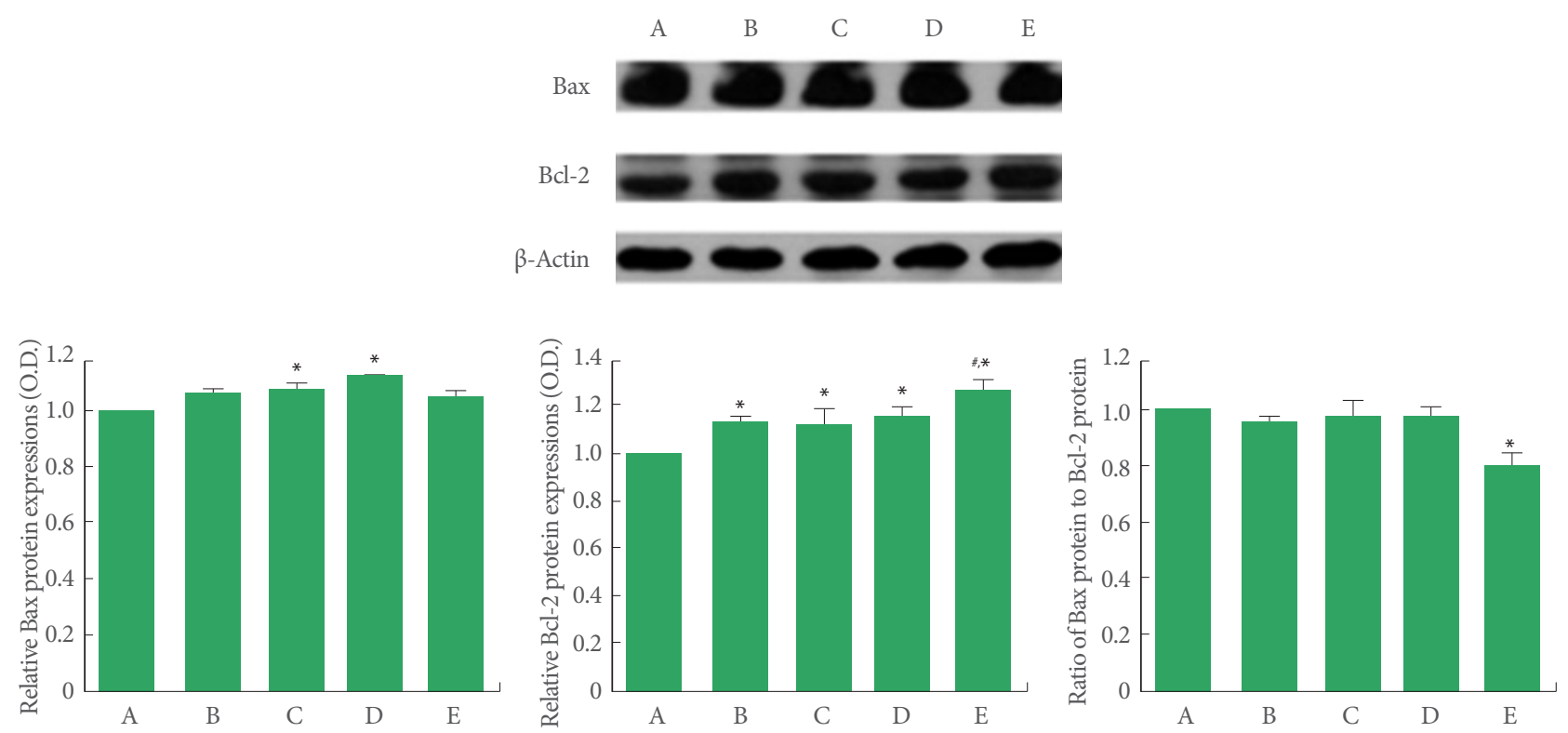

Fig. 6. The effect of dexmedetomidine on the expression of Bax and Bcl-2 in the hippocampus. Upper panel: expression bands of Bax and Bcl-2. Lower panel: relative expression levels of Bax and Bcl-2. A, control group; B, the group in which dexmedetomidine was attached for 2 hours; C, the group in which dexmedetomidine was attached for 6 hours; D, the group in which dexmedetomidine was attached for 12 hours; E, the group in which dexmedetomidine was attached for 24 hours. ${ }^{*} \mathrm{P}<0.05$ compared to the control group. ${ }^{\#} \mathrm{P}<0.05$ compared to the 2-hour dexmedetomidine group.
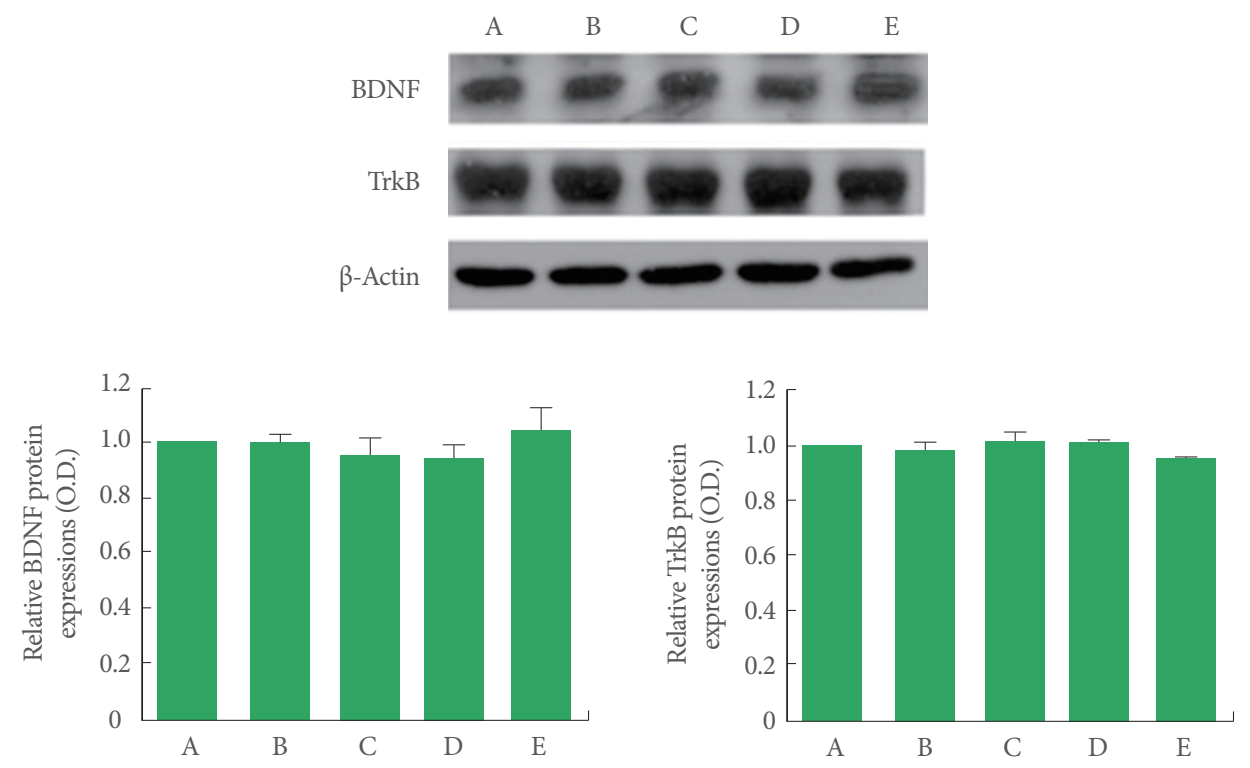

Fig. 7. The effect of dexmedetomidine on the expressions of BDNF and TrkB in the hippocampus. Upper panel: expression bands of BDNF and TrkB. Lower panel: relative expression levels of BDNF and TrkB. A, control group; B, the group in which dexmedetomidine was attached for 2 hours; $C$, the group in which dexmedetomidine was attached for 6 hours; D, the group in which dexmedetomidine was attached for 12 hours; E, the group in which dexmedetomidine was attached for 24 hours. BDNF, brain-derived neurotrophic factor; TrkB, tyrosine kinase B.

cording to the attachment time of the oral mucosa patch. Plasma dexmedetomidine concentrations reached their maximum level at 12 hours after the oral mucosa patch was attached, and then decreased. The results of our study indicate that the oral 
mucosa patch continuously delivered dexmedetomidine into the blood at a constant rate.

Cell proliferation in the hippocampal dentate gyrus is known to be correlated with learning capability and memory function $[14,19]$. Ki-67 is used as a marker for cell proliferation analysis in the hippocampus $[13,20]$. Increments in the number of Ki67-positive cells represent increased cell proliferation, which is associated with the improvement of memory function $[13,14]$. In the present study, the number of Ki-67-positive cells was not changed by attaching the oral mucosa patch, regardless of the attachment time. The present results reveal that dexmedetomidine exerted no significant effect on cell proliferation in the hippocampal dentate gyrus.

Chromatin condensation, cell shrinkage, internucleosomal DNA fragmentation, and apoptotic bodies are the morphological characteristics of apoptosis [21]. TUNEL staining detects DNA fragmentation, representing apoptotic cell death. Caspases are a family composed of 14 cysteine proteases, and they are essential players in the initiation (caspase-2, caspase-8, caspase-9, and caspase-10) and execution (caspase-3, caspase-6, and caspase-7) of the apoptosis process [22]. Upregulation of caspase-3 expression indicates the activation of apoptosis following brain insults $[23,24]$. Increased numbers of TUNEL-positive and caspase-3-positive cells in the hippocampus indicate increased apoptotic neuronal cell death in the hippocampus $[17,24]$. Decreased numbers of TUNEL-positive and caspase-3-positive cells in the hippocampus represent an antiapoptotic effect [17]. In the present study, the numbers of TUNEL-positive cells and caspase-3-positive cells in the hippocampal dentate gyrus decreased as the attachment time of the oral mucosa patch increased. The present results suggest that dexmedetomidine exerted an antiapoptotic effect depending on the attachment time of the oral mucosa patch under normal conditions.

The Bcl-2 family comprises antiapoptotic proteins, including $\mathrm{Bcl}-2$, and proapoptotic proteins, such as Bax. By preventing the release of cytochrome $\mathrm{c}$ from the mitochondria, $\mathrm{Bcl}-2$ exerts an inhibitory effect on apoptosis. Bcl-2 forms heterodimers with the main pro-apoptotic member Bax, at which point it can no longer carry out its protective function [25]. Thus, the ratio of Bax to Bcl-2 is used to determine whether cells undergo apoptosis or not [26]. In the present study, the expression of Bax and Bcl-2 in the hippocampus increased according to the attachment time of the oral mucosa patch; however, the ratio of Bax to Bcl-2 decreased 24 hours after attaching the oral mucosa patch. The present results demonstrate that dexmedetomidine exerted a suppressing effect on apoptosis 24 hours after attaching the oral mucosa patch in normal rats.

BDNF controls neural growth and survival, and BDNF is implicated in learning ability and memory process via its receptor, TrkB $[27,28]$. BDNF enhances hippocampal long-term potentiation, and is a prime candidate for the cellular mechanism of learning, through the action of $\operatorname{TrkB}$ [28]. The upregulation of BDNF and TrkB expression is associated with increased cell proliferation in the hippocampus [29]. Stressful situations have been found to suppress BDNF and TrkB expression in the hippocampus [30]. In the present study, the expression levels of BDNF and TrkB was not changed by attaching the oral mucosa patch, regardless of the attachment time. The present results indicate that dexmedetomidine exerted no significant effect on learning ability or memory-related processes.

In this experiment, we found that a dexmedetomidine oral mucosa patch continuously delivered dexmedetomidine into the blood. An antiapoptotic effect of dexmedetomidine appeared 6 hours after attaching an oral mucosa patch and reached its maximum level 24 hours after attachment. The present study suggests that a dexmedetomidine oral mucosa patch can be used as a convenient tool for sedation and that it may be of therapeutic value due to its antiapoptotic effects under normal conditions.

\section{REFERENCES}

1. Paris A, Tonner PH. Dexmedetomidine in anaesthesia. Curr Opin Anaesthesiol 2005;18:412-8.

2. Jevtovic-Todorovic V, Hartman RE, Izumi Y, Benshoff ND, Dikranian K, Zorumski CF, et al. Early exposure to common anesthetic agents causes widespread neurodegeneration in the developing rat brain and persistent learning deficits. J Neurosci 2003;23:876-82.

3. Duan X, Li Y, Zhou C, Huang L, Dong Z. Dexmedetomidine provides neuroprotection: impact on ketamine-induced neuroapoptosis in the developing rat brain. Acta Anaesthesiol Scand 2014;58:1121-6.

4. Li Y, Zeng M, Chen W, Liu C, Wang F, Han X, et al. Dexmedetomidine reduces isoflurane-induced neuroapoptosis partly by preserving PI3K/Akt pathway in the hippocampus of neonatal rats. PLoS One 2014;9:e93639.

5. Afonso J, Reis F. Dexmedetomidine: current role in anesthesia and intensive care. Rev Bras Anestesiol 2012;62:118-33.

6. Engelhard K, Werner C, Eberspächer E, Bachl M, Blobner M, Hildt $\mathrm{E}$, et al. The effect of the a2-agonist dexmedetomidine and the $\mathrm{N}$ methyl-D-aspartate antagonist $\mathrm{S}(+)$-ketamine on the expression of 
apoptosis-regulating proteins after incomplete cerebral ischemia and reperfusion in rats. Anesth Analg 2003;96:524-31.

7. Dahmani S, Paris A, Jannier V, Hein L, Rouelle D, Scholz J, et al. Dexmedetomidine increases hippocampal phosphorylated extracellular signal-regulated protein kinase 1 and 2 content by an $\alpha 2-$ adrenoceptor-independent mechanism: evidence for the involvement of imidazoline I1 receptors. Anesthesiology 2008;108:457-66.

8. Sanders RD, Xu J, Shu Y, Januszewski A, Halder S, Fidalgo A, et al. Dexmedetomidine attenuates isoflurane-induced neurocognitive impairment in neonatal rats. Anesthesiology 2009;110:1077-85.

9. Sanders RD, Sun P, Patel S, Li M, Maze M, Ma D. Dexmedetomidine provides cortical neuroprotection: impact on anaesthetic-induced neuroapoptosis in the rat developing brain. Acta Anaesthesiol Scand 2010;54:710-6.

10. Kim SE, Ko IG, Kim CJ, Chung JY, Yi JW, Choi JH, et al. Dexmedetomidine promotes the recovery of the field excitatory postsynaptic potentials (fEPSPs) in rat hippocampal slices exposed to oxygen- glucose deprivation. Neurosci Lett 2016;631:91-6.

11. Hwang L, Choi IY, Kim SE, Ko IG, Shin MS, Kim CJ, et al. Dexmedetomidine ameliorates intracerebral hemorrhage-induced memory impairment by inhibiting apoptosis and enhancing brain-derived neurotrophic factor expression in the rat hippocampus. Int $\mathrm{J}$ Mol Med 2013;31:1047-56.

12. Choi IY, Hwang L, Jin JJ, Ko IG, Kim SE, Shin MS, et al. Dexmedetomidine alleviates cerebral ischemia-induced short-term memory impairment by inhibiting the expression of apoptosis-related molecules in the hippocampus of gerbils. Exp Ther Med 2017;13:107-16.

13. Sung YH, Shin MS, Cho S, Baik HH, Jin BK, Chang HK, et al. Depression-like state in maternal rats induced by repeated separation of pups is accompanied by a decrease of cell proliferation and an increase of apoptosis in the hippocampus. Neurosci Lett 2010;470: 86-90.

14. Lee SM, Kim BK, Kim TW, Ji ES, Choi HH. Music application alleviates short-term memory impairments through increasing cell proliferation in the hippocampus of valproic acid-induced autistic rat pups. J Exerc Rehabil 2016;12:148-55.

15. Park JH, Kim SE, Jin JJ, Choi HS, Kim CJ, Ko IG. Pentoxifylline alleviates perinatal hypoxic-ischemia-induced short-term memory impairment by suppressing apoptosis in the hippocampus of rat pups. Int Neurourol J 2016;20:107-13.

16. Roh JH, Ko IG, Kim SE, Lee JM, Ji ES, Kim JH, et al. Treadmill exercise ameliorates intracerebral hemorrhage-induced depression in rats. J Exerc Rehabil 2016;12:299-307.
17. Baek SS, Kim SH. Treadmill exercise ameliorates symptoms of Alzheimer disease through suppressing microglial activation-induced apoptosis in rats. J Exerc Rehabil 2016;12:526-34.

18. Han JH, Kim DO, Yi JW, Park SW, Kang WJ, Choi YK, et al. Dexmedetomidine, a2-adrenoceptor agonist, does not induce apoptosis in the brachial plexus of rats. Anim Cells Syst 2014;18:407-15.

19. Gould E, Beylin A, Tanapat P, Reeves A, Shors TJ. Learning enhances adult neurogenesis in the hippocampal formation. Nat Neurosci 1999;2:260-5.

20. Kee N, Sivalingam S, Boonstra R, Wojtowicz JM. The utility of Ki67 and BrdU as proliferative markers of adult neurogenesis. J Neurosci Methods 2002;115:97-105.

21. Li Y, Chopp M, Jiang N, Zhang Z. G, Zaloga C. Induction of DNA fragmentation after 10 to 120 minutes of focal cerebral ischemia in rats. Stroke 1995;26:1252-7.

22. Reed CJ. Apoptosis and cancer: strategies for integrating programmed cell death. Semin Hematol 2000;37(4 Suppl):9-16.

23. Benchoua A, Braudeau J, Reis A, Couriaud C, Onténiente B. Activation of proinflammatory caspases by cathepsin $B$ in focal cerebral ischemia. J Cereb Blood Flow Metab 2004;24:1272-9.

24. Suh HJ, So SM, Na YG, Ko IG, Kim SE, Sung YH, et al. Neuroprotective effects of tamsulosin on intracerebral hemorrhage. Neural Regen Res 2011;6:2505-10.

25. Kuwana T, Newmeyer DD. Bcl-2-family proteins and the role of mitochondria in apoptosis. Curr Opin Cell Biol 2003;15:691-9.

26. Upadhyay D, Panduri V, Ghio A, Kamp DW. Particulate matter induces alveolar epithelial cell DNA damage and apoptosis: role of free radicals and the mitochondria. Am J Respir Cell Mol Biol 2003;29:180-7.

27. Gomez-Pinilla F, Vaynman S. A "deficient environment" in prenatal life may compromise systems important for cognitive function by affecting BDNF in the hippocampus. Exp Neurol 2005;192:23543.

28. Minichiello L. TrkB signalling pathways in LTP and learning. Nat Rev Neurosci 2009;10:850-60.

29. Hwang DS, Kwak HB, Ko IG, Kim SE, Jin JJ, Ji ES, et al. Treadmill exercise improves memory function depending on circadian rhythm changes in mice. Int Neurourol J 2016;20(Suppl 2):S141-9.

30. Lee SJ, Kim TW, Park HK, Yoon S, You AH, Moon EJ, et al. Postnatal treadmill exercise alleviates prenatal stress-induced anxiety in offspring rats by enhancing cell proliferation through 5-hydroxytryptamine 1A receptor activation. Int Neurourol J 2016;20(Suppl 1):S57-64. 